\title{
LA RUPTURA DE LA NORMA ACENTUAL EN LA POESÍA MODERNISTA: EL EJEMPLO DE JULIO HERRERA Y REISSIG
}

Ángeles Estévez

UNED

En la Gramática de la lengua castellana, de Antonio de Nebrija, al referirse su autor a «los acentos que tiene la lengua castellana», advierte algunos desplazamientos acentuales en la poesía de Juan de Mena, a quien consideraba el más alto poeta de la lengua castellana. Éstas son las palabras del maestro andaluz:

"La segunda regla sea: que todas las palabras de nuestra lengua común mente tienen el acento agudo en la penúltima sílaba, y en las dicciones bárbaras o cortadas del latín, en la última sílaba muchas veces, y muy pocas en la tercera, contando desde el fin; y en tanto grado rehúsa nuestra lengua el acento en este lugar, que muchas veces nuestros poetas, pasando las palabras griegas y latinas al castellano, mudan el acento agudo en la penúltima, teniéndolo en la que está antes de aquélla. Como Juan de Mena:

\section{A la biuda Penelópe I al hijo de Liriópe;}

i en otro lugar:

Con toda la otra mundana machina* !

I Antonio de Nebrija: Gramática de la lengua castellana, estudio y edición de Antonio Ouilis, Madrid, Editora Nacional, 1980, p.138. 
El autor de la primera gramática en lengua romance ya contempló la traslación acentual ${ }^{2}$ en un poeta que tanta influencia ha ejercido ${ }^{3}$, y a quien tanto consideró, hasta el punto de afirmar: "Antonomasia es cuando ponemos algún nombre común por el propio, y esto por alguna excelencia que se halla en el propio más que en todos los de aquella especie; (...) como diziendo el Poeta, entendemos Virgilio y Juan de Mena” ${ }^{4}$. No es de extrañar que Nebrija vaya a tomar la mayor parte de los ejemplos de su Gramática en la obra del poeta cordobés.

Convendria detenerse en las justificaciones que se han dado al desplazamiento acentual en Juan de Mena. Fernando Lázaro escribió: «Cabe decir, por ejemplo, que Mena (...) acentúa Penelópe a causa de la tendencia general del castellano a los paroxítonos; o que escribe cástalo y no castalio por imitación de adjetivos latinos del tipo Thessaulus, Italus (...); son, todas, aserciones plausibles, pero no explican la razón última de por qué prefirió el cordobés tales soluciones. $Y$, menos aún, por qué, en otros lugares, el propio Mena empleó (...) Penélope (...) y castalio (...) contrariando así las justificaciones anteriores» 5 .

Pero el desplazamiento acentual carece de sentido en sí, es decir, en la misma palabra que lo soporta, por eso - muy atinadamente- Antonio de Nebrija no nos cita la palabra sola sino el hemistiquio o el verso completo. Desde esta perspectiva, Foulché-Delbosc estudió la estructura del dodecasílabo del Laberinto ${ }^{6}$ y encontró que estaban divididos en dos hemistiquios, que pueden tener diferente número de sílabas cada uno, «y que, a su vez, tienen generalmente dos acentos, o bien uno solo, aunque esto es más raro (...). El hemistiquio de un solo acento puede terminar en forma oxítona o paroxítona. Sólo halla estas dos combinaciones:

$$
\begin{aligned}
& x \quad u \quad u v-u \text { en virtud diversa }(66,2) \\
& y \quad u \quad u v-\text { menos en la lid }(4,3) »
\end{aligned}
$$
p. 70 .

${ }^{2}$ Antonio Quilis considera dicha traslación como licencia poética, en estudio citado,

${ }^{3}$ José Manuel Blecua dedica una parte de su prólogo a «La admiración por Juan de Mena», en El laberinto de Fortuna o Las Trescientas, de Juan de Mena, Madrid, EspasaCalpe, 1960 , p. XCI-CII.

Antonio de Nebrija: Op. cit., p. 222.

5 Fernando Lázaro Carreter: «La poética del Arte Mayor castellano», p. 77, en Estudios de poética, Taurus, Madrid, 1976, p. 75-III. Este trabajo fue publicado en Studia Hispanica in Honorem Rafael Lapesa. T. I, Madrid, 1972, pp. 343-378.

Encontramos un buen resumen del estudio de Foulché-Belbosc en el prólogo citado de Jose Manuel Blecua, pp. LXXXIV-XCI.

José Manuel Blecua: prólogo citado, p. LXXXV. 
Lázaro Carreter ha observado que estos hemistiquios con un solo ictus es enturbiar el hallazgo, y añade: «de postular otro ictus anterior, éste debía recaer sobre la primera sílaba de vírtud y sobre la segunda de menós, deformándolos prosódicamente» ${ }^{8}$. Pues bien, estos cambios acentuales ya fueron parcialmente reseñados en nuestra primera Gramática. El ritmo puede desplazar al acento, como hemos visto. «Se trata de un artificio eficacísimo para cualquier poética que se base en un profundo distanciamiento entre la lengua del verso y el idioma común» ${ }^{9}$. Es también Lázaro Carreter quien nos recuerda que las traslaciones acentuales ya ocurrían en la poesía medieval, porque el ritmo del poeta y del lector va gobernado por lo que Jakobson ha llamado 'modelo de verso'. "Los primeros beneficiarios de esta subrogación del acento fueron los neologismos y los nombres propios, cuya rareza les permitia figurar en el hemistiquio con su acento desplazado o no, sin sorpresa para nadie» ${ }^{10}$. Estas traslaciones son, sin embargo, las más llamativas y a ellas se refirió Nebrija al señalar Penelópe o machína en Mena.

Me referiré al desplazamiento acentual en la poesía de Julio Herrera y Reissig, pero sólo estudiaré dos casos: el que se refiere a las rimas y al primer hemistiquio de los alejandrinos. He elegido al poeta uruguayo porque es considerado como el más alto representante del modernismo canónico. He realizado también el mismo estudio - aunque en estos casos sólo referido a las rimas- en toda la poesía de Rubén Darío "1; en Castalia bárbara ${ }^{12}$, de Ricardo Jaimes Freyre; en Los crepúsculos del jardín ${ }^{13}$, de Leopoldo Lugones. La elección de estas obras obedece a causas diferentes que expondré a continuación.

Elegir a Rubén parece obvio, al tratarse del "pontífice» del Modernismo. Por otra parte, está demostrada la influencia de Darío en los primeros poemas modernistas de Herrera y Reissig. Además, se ha estudiado - aunque muy parcialmente- la dislocación acentual en el autor de $\mathrm{Azul}^{14}$.

${ }^{*}$ Fernando Lázaro Carreter: artículo citado, pp. 79-80.

Ibidem, p. 82.

10 Ibidem, pp. 81-82.

11 Rubén Dario: Poesías completas, edición, introducción y notas de Alfonso Méndez Plancarte, aumentada con nuevas poesías y otras adiciones por Antonio Oliver Belmás, Madrid, Aguilar, 1967, 10." ed.

12 Ricardo Jaimes Freyre: Castalia bárbara, prólogo de Juan Siles Guevara, La Paz, Ministerio de Educación y Cultura, Fondo Nacional de Cultura, 1970.

${ }_{13}$ Leopoldo Lugones: "Los crepúsculos del jardín (1905)», en Obras poéticas completas, pp.105-188, prólogo de Pedro Miguel Obligado, Madrid, Aguilar, 1959, 3." ed.

14 Antonio Oliver Belmás: "La dislocación acentual en la poesía de Rubén Darío», en Cuadernos Hispanoamericanos, Madrid, agosto-septiembre 1967, pp. 405-409, n." 212213, dedicado a Rubén Darío. En este número se publicó, entre otros, un trabajo de Gerardo Diego ("Ritmo y espíritu en Rubén Dario») y otro de Federico Sopeña («Rubén 
Antonio Oliver Belmás ha señalado cuatro desplazamientos en la poesía de Dario: libelúla, Condóres, Fatíma y Medicís, y termina así su trabajo: "Con estos cuatro ejemplos no hemos agotado, indudablemente, todos los casos de desplazamiento acentual en el verso de Rubén Darío, pero quizá hemos subrayado, eso sí, los más significativos» 15 .

Si se puede argumentar que en la poesía de la baja Edad Media el estado vacilante de la lengua castellana pudo favorecer el desplazamiento acentual, lógicamente este argumento no es válido para el Modernismo. Habrá que buscar otras razones.

Condorres aparece en el poema «En el álbum de Pedro Nolasco Préndez», fechado en Santiago de Chile (marzo de 1887). Quizá estemos ante el primer desplazamiento acentual de Rubén, que refiere en la citada composición la comparecencia del poeta argentino Olegario Víctor Andrade (1839-1882) ante Apolo, y menciona una de sus composiciones más célebres «El nido de Cóndores»-, que trata sobre la repatriación de los restos de San Martín. Andrade se queja del poco aprecio a su poesía y Apolo responde:

Fui contigo dulce y blando...

¡Hasta ganas me están dando

de tirarte las orejas!

Te quejas, cuando de flores

ofrecen guirnaldas hermosas

a tu Atlántida grandiosa

y a $u$ u Nido de Condóres.

16.

La ironía abarca todo el poema, y para lograrla, Rubén echa mano no sólo de este desplazamiento sino de la utilización de arcaísmos («do»). Pero quizá la ironía afecte también a la mitología política e incluso patriótica americana: los altos, esdrújulos y conocidísimos «cóndores» del poema heroico de Andrade son los llanos condóres de Rubén Darío, para quien el ave andina era símbolo de la victoria, como leemos en «Marcha triunfal»:

Darío en la música») para llegar a conclusiones opuestas en lo que se refiere a lo musicalrítmico.

${ }^{15}$ Ibidem, p. 409.

in Rubén Darío: Poesías completas, ed. cit., p. 870. 
Dejando el picacho que guardan sus nidos, tendiendo sus alas enormes al viento, los cóndores llegan. ¡Llegó la victoria!

17

El duende Puck es protagonista de dos poemas de Darío («los regalos de Puck» y «¿Dónde estás?») en los que hay dos desplazamientos: libelúla y Fatíma, respectivamente ${ }^{18}$. Ambas composiciones son anteriores a 1893 y domina en ellas el aire festivo, aunque la segunda «parece inspirada en la muerte de "Stella" (...), la primera esposa de Rubén» ${ }^{19}$. En cuanto a Medicís, es de «En el Luxembourg», y está fechado en octubre de $1907^{20}$.

He encontrado en Rubén Darío otros ejemplos de desplazamiento que considero importantes ${ }^{21}$. Antes de analizarlos, conviene recordar que en ningún caso opino que se trata de descuidos, pues Rubén conocía muy bien la acentuación, como lo demuestra, por ejemplo, con la palabra cenit $^{22}$, que aparece así correctamente acentuada y no el tan usado cénit.

Exódo lo encontré en «Revelación» ${ }^{23}$, que es uno de los poemas más importantes de El canto errante, tal vez porque "en esta poesía es misteriosamente panteísta» ${ }^{24}$, o quizá por la sabia combinación de mitos encarnados en el propio poeta. Éste es el final de «Revelación»:

$Y$ con la voz de quien aspira y ama,

clamé: ¿¿Dónde está el dios que hace del lodo

17 Ibidem, pp. 646-647.

18 Ibidem, pp. 943 y 947.

19 Antonio Oliver Belmás: art. cit., p. 407.

20 Rubén Darío: Poesías completas, ed. cit., pp. 1.018-1.020.

21 Para este recuento utilizo la citada edición de Rubén Dario.

22 Cenit lo encuentro dos veces (ed. cit., pp. 591 y 739, respectivamente). El primero pertenece al poema $*$ Sinfonía en gris mayor» y a «Hondas» el segundo. Según Marasso (Rubén Dario y su creación poética, La Plata, 1934, p. 95), el título es una variante de la *Sinfonía en blanco mayor* de Théophile Gautier. La coincidencia de títulos e intenciones entre Rubén y Gautier fue señalada por José Enrique Rodó en su excelente ensayo *Rubén Darion, en Obras completas. Vol. II, Montevideo, 1956, pp. 90-92. Rodó vio en 1900 el origen de la sinestesia en castellano. José Asunción Silva (fallecido en 1896) parodia la sinestesia en castellano y los ambientes de Rubén en su poema *Sinfonía color de fresas con leche», en Antología crítica de la poesía modernista hispanoamericana, prólogo de J. Olivio Jiménez, Madrid, Hiperión, 1985, p. 165.

${ }^{23}$ Rubén Darío: Poesías completas, ed. cit., pp. 712-713.

24 Arturo Marasso: Op. cit., pp. 268-270. 
con el hendido pie brotar el trigo,

que a la tribu ideal salva en su exódo?»

$Y$ oi dentro de mí: «Yo soy contigo,

$y$ estoy en ti y por ti, yo soy el Todo» 25 .

Otras veces, el desplazamiento acentual ocurre en neologismos propios de Darío, y su intención es puramente festiva. Retrocedamos a una composición de circunstancias, «Balada a Leopoldo Díaz para que tome por cancillera a una de las nueve Musas» ${ }^{26}$, que pertenece a la sección «Bajo el Sol Argentino». El poema está fechado en Buenos Aires en 1897, dedicado a Leopoldo Díaz que parte para Europa como cónsul. Termina así:

con que has vencido la suerte ingrata;

y en tu carrera de diplomáta

¡lleva una Musa por cancillera!

Si antes el desplazamiento iba reforzado por el arcaísmo, ahora lo vemos acompañado de un femenino inusual. La intención festiva la encuentro también a lo largo de una décima, fechada en Managua en 1893. Su título es significativo: «Décima de pie forzado acabando en "patio"», y dice:

No entiende de acentos Pablo, pues cuando dice una frase

forma un requiescat in pace

que es capaz de darse al diablo.

Si con él converso o hablo,

por batió me dice batio

y por combatio, combatio.

Un día me sublevé

$y$ por poco no arranqué

el rosal que está en su patio ${ }^{27}$.

25 Marasso no comenta este desplazamiento acentual.

26 Ibidem, pp. 989-990.

27 Ibidem, p. 959. Debajo del título leemos: «En un juego de rimas con Manuel Maldonado y Desiderio Fajardo Ortizx. 
Frente al ejemplo festivo citado, encontramos un ejemplo importante y final en el poema «A Francisca», fechado en París (21 de febrero de 1914). La composición figura entre las más conocidas de Dario, quizá porque vuelca en ella su espíritu. El desplazamiento se da en los cuartetos finales y afecta a acompañamé, que se repite dos veces. Así termina el poema:

Seguramente Dios te ha conducido para regar el árbol de mi fe. ¿Hacia la fuente de noche y olvido, Francisca Sánchez, acompañamé! ${ }^{28}$.

Rubén Darío ha utilizado los desplazamientos acentuales al final de verso en todas las etapas de su poesía y con todas las intenciones: desde la cómica a la plenamente modernista o en el final de «A Francisca», cuando el poeta sabía que ya se encaminaba a la «fuente de noche y de olvido».

Ricardo Jaimes Freyre fue el segundo autor al que me dirigí. Había leído yo que "su primer libro -Castalia bárbara [1899 y no 1897 como escribe Anderson]_ fue un laboratorio experimental de ritmos. El ritmo por el ritmo" ${ }^{29}$. Además, Ricardo Jaimes no sólo es maestro del modernismo con el poemario citado, sino cofundador - junto con Darío- de la célebre Revista de América (1894) de Buenos Aires y de la publicación modernista Revista de Letras y Ciencias Sociales (1904-1907) de Tucumán, donde publica fragmentariamente Las leyes de la versificación castellana, que aparece como volumen en $1912{ }^{30}$. Jaimes confiesa en el prólogo de sus Leyes...: «Considero definitivamente enterrada la

${ }^{28}$ Ibidem, pp. 1.082 y 1.083 . Ángel Rama habla de la «inmediatez dramática tan vigorosa que ha enmascarado un poema cargado de flojedades retóricas (...) que se han desvanecido ante el impacto emocional de esta brusca confesión angustiada», en Rubén Darío y el modernismo, Ediciones de la Biblioteca de la Universidad Central de Venezuela, Caracas, 1970, p. 110. Rama se refiere a los versos: «multiplicaste pétalos de lirio/ y refrescaste la hoja de laurel».

Sobre acompañamé: peculiaridad rioplatense (muy utilizada en la poesía gauchesca). Vid. Eleuterio Tiscornia: La lengua de «Martín Fierro», T. III, de la Biblioteca de Dialectología Hispanoamericana, Buenos Aires, 1930. Eliseo Diego se ha referido a la eficacia emotiva de acompañamé, en "Rubén Darío», Casa de las Américas, año VII, n." 42, pp. 80-81, La Habana, mayo-junio de 1967.

Enrique Anderson Imbert: Historia de la literatura hispanoamericana, T. I., México, Fondo de Cultura Económica, 1967, 6." ed. (1. “: 1954), pp. 381-382.

30) Ricardo Jaimes Freyre: Poemas. Leyes de la versificación castellana, México, Aguilar, 1964, prólogo de Antonio Castro Leal. 
hipotesis de sílabas largas y breves y atribuyo sólo al acento la virtud de generar el ritmo" ${ }^{31}$. Al considerar el acento como el espiritu del ritmo, difícilmente podría ignorar Jaimes el desplazamiento acentual al final de verso:

«Es de notar que las palabras sobresdrújulas (que en general deben ser desterradas de la versificación) no se encuentran en las mismas condiciones, pues sus tres últimas sílabas no son embebidas en la pausa métrica. En las raras ocasiones en que han sido usadas al final del verso se ha acentuado su última sílaba:

\section{Seguid buscandomeló» ${ }^{32}$.}

Rechaza el desplazamiento acentual, pues, y tolera como licencia la acentuación rítmica en palabras átonas, en palabras que no tienen acento prosódico» ${ }^{33}$, en clara alusión a Rubén Darío que la usó sistemáticamente. Ricardo Jaimes se preocupa fundamentalmente de los períodos prosódicos, y no es partidario de la combinación artística de períodos diferentes, que él mismo ensayó en Castalia bárbara. «No han tenido continuadores - añade-, lo que es un rudo argumento en contra» ${ }^{34}$. No es de extrañar, pues, que en Castalia bárbara no aparezca ningún desplazamiento acentual.

Leopoldo Lugones fue otro modernista donde busqué desplazamientos acentuales. Me limité a Crepúsculos del jardín porque esta obra ha sido señalada por varios críticos como posible fuente de algunos poemas de Julio Herrera. Encontré en Crepúsculos... otras influencias del poeta argentino sobre el uruguayo: desde rimas («lerdas/cuerdas» -p. 184-) a neologismos («ritmar», por ejemplo, que está ya en Darío) o sintagmas poéticos («dedos de rosa» -p. 186-). Sin embargo, desplazamientos acentuales a final de verso he visto sólo uno, y está en el poema "Camelia»:

Cómo se llama el corazón lo augura:

-Clelia, Eulalia, Clotilde- algún pristíno

nombre con muchas eles, como un fino

cristal, todo vibrante de agua pura.

31 Ibidem, p. 167.

Ibidem, p. 192.

Ibidem, p. 235.

34 Ibidem, p. 211.

35 Leopoldo Lugones: Op. cit., p. 126. 
Creo que el desplazamiento en pristíno colabora en la elaboración de la suntuosidad modernista decadente que refleja «Camelia».

Julio Herrera y Reissig es el poeta modernista que emplea con más generosidad los desplazamientos acentuales. A pesar de ello, en los estudios dedicados a su poesía este fenómeno ha sido ignorado, incluso en la que sigue siendo la obra global más importante sobre su estilo, la tesis de Yolando Pino Saavedra ${ }^{36}$. Por mi parte, aislé algunos casos en mi Memoria de licenciatura. En ese trabajo, escribí entonces: «Sólo dos veces usa el [adjetivo] esdrújulo en las rimas de "Desolación absurda [poema clave en la evolución de Julio Herrera], y en una ocasión desplaza el acento para evitarlo: cefiros ${ }^{37}$. Y en otro lugar de mi trabajo escribí: «He tomado "Misa bárbara" porque creo que este soneto en endecasílabos representa bien varias características de la poesía de $\mathrm{He}$ rrera: desplazamiento acentual al final de verso (Eufrátes) „, aunque esta palabra admite la doble acentuación ${ }^{38}$. Me he permitido la autocita porque por primera vez se trataba el desplazamiento acentual en la poesía del autor de Los Peregrinos de Piedra.

Encontramos en cefiros el primer ejemplo, y pertenece al poema « Naturaleza!» publicado en $1899{ }^{39}$. Dice así:

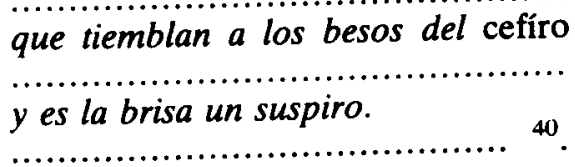

«Naturaleza!» se debe incluir en la producción premodernista herreriana. Pero en «Desolación absurda» - superado ya el modernismo inicial- encontramos:

36 Yolando Pino Saavedra: La poesía de Julio Herrera y Reissig: Sus temas y estilo, Santiago de Chile, 1932.

37 Angeles Estévez: El léxico modernista de Julio Herrera y Reissig. Memoria de licenciatura (inédita), Universidad Complutense de Madrid, julio de 1984, p. 28.

Ibidem, p. 68.

39 Antonio Seluja: Julio Herrera y Reissig. Vida y obra, Montevideo, 1984, pp. 59-60.

40 Julio Herrera y Reissig: Poesias completas y páginas en prosa, edición, estudio preliminar y notas de Roberto Bula Píriz, Madrid, Aguilar, 1961, p. 235.

Mario Álvarez Rodríguez ha visto claramente la función de «Naturaleza» en la obra herreriana: «Apunta (...) un incipiente don verbal e imaginístico. (...) «Naturaleza (...) es como un borrador romántico y torpe de la obra ulterior», en Poesías de J. Herrera y Reissig, Montevideo, Ediciones de la Banda Oriental, 1975, p. 9. 
Noche de tenues suspiros

Platónicamente ilesos

Vuelan bandadas de besos

$Y$ parejas de suspiros

Ebrios de amor los cefíros ${ }^{41}$.

Idénticos cefíros en ambos poemas, pero con diferente intención: en el primero, el equilibrio; la ironía, en el segundo.

También a «Desolación absurda» pertenece otro desplazamiento, esta vez en nombre propio: Mendelssohn. Se ha escrito mucho sobre los conocimientos musicales de Julio Herrera, pero, nos quedemos de un lado u otro, el poeta uruguayo no ignoraba la prosodia del músico alemán. Al desplazar el acento, insiste en lo grotesco de la manida estampa romántica:

(Es media noche). Las ranas

Torturan en su acordeón

Un «piano» de Mendelssóhn

Que es un gemido de ranas

Encontramos otro desplazamiento en uno de los poemas más elaborados del poeta uruguayo, «Los ojos negros» ${ }^{43}$. Dice:

Fuegos fatuos que lucieron

En la Reina de Sabá ${ }^{44}$

\footnotetext{
41 Julio Herrera y Reissig: Las Pascuas del Tiempo, T. IV, Montevideo, Bertani, 1913, p. 67.

42 lbidem, p. 72.

43 Herrera y Reissig publicó la primera versión de este poema en La Revista (año I, T. II, n. 11 , pp. 460-465, Montevideo, junio 1900). El poema se llamó entonces *Psicologia de unos ojos negros». En el Ms. 14 A III 21 F 5 del Archivo Julio Herrera y Reissig - Departamento de Investigaciones Literarias de la Biblioteca Nacional de Montevideoel título es \&Los ojos negros», y en él aparece ya la décima que contiene el desplazamiento Saba. Por fin, en la edición Bertani, el poema se llamó «Los ojos negros de Julieta*.

"Rubén Darío también empleó Saba en rima, pero sin desplazamiento: «alaba/ Sabax, en Poesias completas, ed. cit., p. 52.
} 
«Recepción» es también un poema muy elaborado, y el desplazamiento aparece en los versos añadidos en la última versión, igual que ocurre con el ejemplo citado antes. Esta vez se trata de una palabra perteneciente a la liturgia católica, Pentescóstes, que el poeta conocía muy bien. Julio Herrera eligió dicho poema para pórtico del único libro que ordenó personalmente. Citamos los versos donde está incardinado:

Todo se inspira. Los Númenes

Trasudan su Pentecóstes;

Se exhalan a Diana, rubios

Muezines, los girasoles. 46.

El afán parnasiano de «Recepción» induce a Herrera a otro desplazamiento:

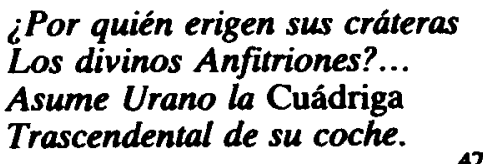

En 1906 -aunque parece que su composición es de tres años antes - se publica en La Democracia de Montevideo el poema *La vida», que luego dio título al tomo $\mathrm{V}$ de las obras completas en la edi-

4s Julio Herrera y Reissig: La vida y otros poemas, Obras completas, T. V., Montevideo, Bertani, 1913, p. 52.

46 Julio Herrera y Reissig: Los Peregrinos de Piedra, Montevideo, Bertani, 1909. p. 11. Rubén Dario expresó con palabras precisas el parnasianismo del poema: «Recepción», una alta columna de mármol en que estuviesen grabados figuras, personajes, grupos mitológicos, en armoniosa confusión». Vid. Antonio Seluja, op. cir., p. 168.

17 Ibidem, p. 14. 
ción de Bertani. Se trata de una de las composiciones más célebres de Julio Herrera, no sólo por sus versos sino por las notas que añadió a pie de página el propio poeta para intentar explicar los elementos herméticos de la composición. Leemos:

Tempestuoso de albatrós
Iba el audaz palafrén
Terrible y congestionado
Por el Enigma, y yo en pos!
....................................

Como Rubén Darío, Julio Herrera dijo exódos, y lo rimó igual en el soneto «Las madres» de Los Peregrinos...:

Y a grave paso acuden, por los senderos todos, Gentes que rememoran los antiguos exódos. 49

Una sección muy conocida de la poesía herreriana está constituida por «Sonetos vascos». Dentro de la misma está «El mayoral», compuesto en plena madurez de su autor. Dice asi su terceto final:

Se recela un instinto algo terco de cabra...

$Y$ soslaya sus ojos de mastin bravo y noble

El orgullo que roe la tristeza cantábra ${ }^{50}$.

Por primera vez termina un poema con un desplazamiento, simbolizando en él y en su rima el desagrado ante un pretendido mundo bucólico.

48 Julio Herrera y Reissig: La vida y..., ed. cit., p. 24. En el Ms. 14 A III 20 F 2 del archivo herreriano citado en la nota 43 , el poeta acentuó albatrós.

49 Julio Herrera y Reissig: Los Peregrinos..., ed. cit., p. 64.

50 Este soneto fue publicado en El Diario Español de Buenos Aires (17 de mayo de 1908 ), junto con *El granjero* y *Vizcaya*. 
En otro soneto vasco - «La trilla» encontramos un nuevo desplazamiento que el propio poeta acentuó cuidadosamente en el manuscrito para distinguirlo, por más que dicho acento sea superfluo. Lógicamente, los editores no acentuaron vizcáina, excepto Bula Píriz que «enmendó» al poeta, y leyó vizcaína. Herrera la rimó con azotáina, Váina y dulzáina, así mismo acentuadas por su autor.

«La Torre de las Esfinges» es la composición más comentada de nuestro poeta, porque es la más hermética y, al mismo tiempo, es considerada como la más vanguardista. Ahí encontramos tres desplazamientos, sin contar metempsicósis, al que nos referiremos luego. En primer lugar, destaquemos Schumánn:

Ladra en un perro Satán,

$Y$ un profesor rascahuesos

Trabuca en hipos aviesos

El Carnaval de Schumánn ${ }^{51}$.

Queda fuera de toda duda que el autor de Los Peregrinos... conocía la correcta acentuación, pero la quiere romper y convierte en oxítono el nombre del músico como vemos en el Ms. 14 A IV 41 F 9. Sin embargo, en otras ocasiones prefiere Schúmann:

Las lejanias se ahuman

Hacia confines aciagos,

$Y$ todo: montes y lagos,

Se congestiona de Schúmann.

O bien:

Bien cupieran en tu joven

Abril, mis horas que abruman,

\footnotetext{
s1 Julio Herrera y Reissig: Los Peregrinos..., ed. cit., p. 119. 24.

52 Julio Herrera y Reissig: «Poema violeta», en Las lunas de oro, Montevideo, 1913, p.
} 
Como un dolor de Beethoven En un ensueño de Schúmann.

También en el manuscrito citado antes está cauchú. Se diría que el poeta sigue las instrucciones de Andrés Bello, que en su Arte métrica escribio: «Las asonancias agudas me parecen avenirse mejor con el estilo jocoso (...) La en $u$ es la más difícil de las agudas y de todas las asonancias, y se adapta decididamente a lo burlesco" 54 . Herrera, pues, convierte en grotesco su propio mundo modernista:

Haz que entre rayos celebre

Su aparición Belcebú,

$Y$ tus besos de cauchú

Me sirvan sus maravillas

Al modo que las pastillas

Del Hada Pari-Banú!

En el mismo poema, leemos:

Un gato negro a la orilla

Del cenador de bambú,

Telegrafía una cu

A Orión que le signa un guiño

$Y$ al fin estrangula un niño

Improntu hereje en miaú!

56

Pasemos por alto metempsicósis, también de «La Torre...», su gran p. 344.

53 Ibidem, p. 30. Otro ejemplo en p.13: «Schúmann/bruman».

34 Andrés Bello: Ortología y métrica, Madrid, Imprenta y Fundición de M. Tello, 1890,

55 Julio Herrera y Reissig: Los Peregrinos..., ed. cit., p. 122.

$\$ 6$ Ibidem. p. 131. 
poema final, porque admite doble acentuación, aunque Julio Herrera prefiere rimarla con neurosis ${ }^{57}$.

Citaremos ahora un caso peculiar: resedá:

Todo flota en un dormido

Ambiente de más Allá...

Y la tarde en tu vestido

Se embriaga de resedá...

Herrera dice resedá, tal como se pronuncia en América. José Asunción Silva escribe:

Tu cuerpo de veinte años entre la roja seda

Tus frescuras de virgen y tu olor de reseda...

Resulta entonces que el llamado «modernista europeizante» - Julio Herrera y Reissig - prefiere - como tantas otras veces - la versión americana.

El Ms. 14 A IV 41 F 2 del archivo herreriano de Montevideo es uno de los muchos diccionarios de rimas de nuestro poeta. En las rimas en -ama puso: inflama, almagama - luego dudó, y después de varias rectificaciones escribió amalgama-, a continuación: escama, mojama (con el significado al margen), proclama, pentagrama, soflama... Evidentemente, Herrera prefiere pentágrama, aunque aquí también hay que echar mano de sus conocimientos musicales: conocía la doble posibilidad acentual del término. Pero ¿respeta la acentuación de su diccionario a la hora del poema? Leemos en su soneto «La cátedra»:

57 Ibidem, p. 122.

58 Julio Herrera y Reissig: «Poema violeta», en Las lunas de oro, Montevideo, Bertani, 1913, p. 14.

39 José Asunción Silva: «Poeta, di paso», en Antologia crítica..., ed. cit., p. 147. 
El Astrónomo enuncia todo un óleo erudito, El explica el pentágrama del Arcano Infinito

Imposible leer aquí pentagráma, como hace Alicia Migdal en su edición, pues en este caso el verso tendría quince sílabas. Pero creemos que no es la economía verbal la que dicta el desplazamiento, sino el juego de esdrújulos, que son una constante en sus sonetos.

Diremos para terminar que Julio Herrera emplea abundantemente los desplazamientos acentuales tanto en la rima como en interior de verso, aunque aquí hemos estudiado casi exclusivamente los finales. Siguiendo a Jakobson, podemos decir que también en Herrera y Reissig el ritmo del poeta y del lector se ha gobernado por el «modelo de verso». Éste sería el caso del último ejemplo citado. Pero los más importantes se pueden inscribir en la intención herreriana de burlarse del modernismo, de oponerse a la armonia dariana, de manifestar -una vez más- su desagrado ante el mundo.

60 Julio Herrera y Reissig: Los Peregrinos..., ed. cit., p. 97. 